\title{
Comunidad de parásitos metazoarios de la charra Cichlasoma trimaculatum en la laguna de Tres Palos, Guerrero, México
}

\author{
Metazoan parasite community in the three-spot cichlid Cichlasoma trimaculatum from Tres \\ Palos Lagoon, Guerrero, Mexico
}

\author{
Juan Violante-González ${ }^{1 *}$, Ma. Leopoldina Aguirre-Macedo² y Agustín Rojas-Herrera ${ }^{1}$ \\ ${ }^{1}$ Unidad Académica de Ecología Marina, Universidad Autónoma de Guerrero, Gran Vía Tropical 20, Fracc. Las Playas, 39390 Acapulco, Guerrero, \\ México. \\ ${ }^{2}$ Centro de Investigación y Estudios Avanzados-Instituto Politécnico Nacional (CINVESTAV-IPN) Unidad Mérida, Carretera Antigua a Progreso Km. \\ 6. Apartado postal 73, Cordemex, 97310 Mérida, Yucatán, México. \\ *Correspondencia: viojuang@yahoo.com.mx
}

\begin{abstract}
Resumen. Entre abril de 2000 y noviembre de 2002 se examinó la composición de la comunidad de parásitos metazoarios de la charra, Cichlasoma trimaculatum a partir de muestras temporales. Fueron recuperadas 12 especies de parásitos (40 969 individuos) de 231 hospederos examinados; 10 helmintos: Ascocotyle (Phagicola) longa, Austrodiplostomum compactum, Cladocystis trifolium, Clinostomum complanatum, Crassicutis cichlasomae, Posthodiplostomum minimum, Pseudoacanthostomum panamense, Neoechinorhynchus golvani, Southwellina hispida y Contracaecum sp., y 2 crustáceos: Argulus sp. y Ergasilus sp. La comunidad se caracterizó por un número mayor de parásitos generalistas y pocos especialistas de cíclidos, además por ser pobre en especies. De las 5 especies comunes dentro de la comunidad, 4 presentaron variación temporal en su dinámica de infección a lo largo del tiempo, la cual fue asociada con cambios ambientales generados durante las temporadas de secas y lluvias. La variación en la dinámica de infección de las especies comunes fue capaz de generar cambios en la estructura, tanto en el nivel de componente como en el de infracomunidad; sin embargo, no se observó un patrón claro, lo que indica que la comunidad puede ser poco predecible, como se sugiere para otras comunidades de parásitos de peces dulceacuícolas.
\end{abstract}

Palabras clave: Ciclidae, Digenea, comunidad componente, infracomunidad, variación temporal.

\begin{abstract}
We analyzed metazoan parasite community composition in the three-spot cichlid Cichlasoma trimaculatum using seasonal samples taken between April 2000 and November 2002 from the Tres Palos Lagoon, Guerrero, Mexico. A total of 231 hosts were examined. Of the 12 parasite species recovered (40,969 individuals), 10 were helminths: Ascocotyle (Phagicola) longa, Austrodiplostomum compactum, Cladocystis trifolium, Clinostomum complanatum, Crassicutis cichlasomae, Posthodiplostomum minimum, Pseudoacanthostomum panamense, Neoechinorhynchus golvani, Southwellina hispida, and Contracaecum sp. The remaining 2 were the crustaceans Argulus sp. and Ergasilus sp. The community was generally poor in number of parasite species, and characterized by a high number of generalist parasites and fewer cichlid specialists. Four of the 5 common parasite species exhibited seasonally variable infection dynamics associated with environmental differences between the dry and rainy seasons. This variation in the dynamics of infection in the common parasite species generated changes in community structure over time. Clear patterns were not observed, however, indicating that this community has low predictability, as has been suggested for other parasite communities from freshwater fish.
\end{abstract}

Key words: Cichlidae, Digenea, component community, infracommunity, temporal variation.

\section{Introducción}

Las comunidades de parásitos de cíclidos del sureste de México son sin duda las más estudiadas, tanto desde un punto de vista cualitativo como cuantitativo (Salgado-

Recibido: 28 marzo 2007; aceptado: 17 diciembre 2007
Maldonado, 1993; Pineda-López, 1994; Vidal-Martínez, 1995; Salgado-Maldonado y Kennedy, 1997; SalgadoMaldonado et al., 1997; Vidal-Martínez et al., 1998; Vidal-Martínez y Kennedy, 2000; Salgado-Maldonado et al., 2001; Vidal-Martínez et al., 2001). Los estudios realizados en lagunas costeras de los estados de Tabasco, Campeche y Yucatán indican que las comunidades de parásitos de cíclidos son ricas y diversas en especies y 
están constituidas en mayor grado por especialistas. Esta región se distingue por presentar la máxima riqueza de especies de cíclidos nativos; de unas 70 identificadas en todo el país, 47 se encuentran distribuidas en el sureste neotropical (Pineda-López, 1994).

El estado de Guerrero se caracteriza por su gran potencial acuícola formado por unas 10 lagunas costeras, de las cuales sobresale la de Tres Palos (99 47' O; $16^{\circ} 48^{\prime} \mathrm{N}$ ), tanto en extensión $\left(55 \mathrm{~km}^{2}\right)$ como en recursos pesqueros (900 ton/año) (SAGARPA, 2000). En esta laguna, la comunidad ictiológica está constituida por 38 especies agrupadas en 18 familias y 30 géneros (Yáñez-Arancibia, 1978). No obstante esta gran diversidad de especies, sólo existen 2 de cíclidos: una introducida (Oreochromis sp.) y otra nativa (Cichlasoma trimaculatum), conocida localmente como charra.

Cichlasoma trimaculatum reviste importancia económica dado que se consume localmente. Se han realizado algunos estudios tendientes a determinar la factibilidad de su cultivo (Violante-González, 1995) y sobre su parasitofauna en el medio natural (ViolanteGonzález, 2006; Violante-González y Aguirre-Macedo, 2007; Violante-González, et al.m., 2007), aspecto muy importante si en un futuro el cíclido es considerado para fines de cultivo.

Por otra parte, en ecología de comunidades de parásitos, un aspecto poco estudiado es si las comunidades presentan o no una variación temporal en su estructura y composición de especies debida a cambios en los factores ambientales a lo largo del año. Los resultados que se han obtenido indican que tienden a presentar cambios temporales, los cuales se asocian con alteraciones ambientales propiciadas por el ciclo estacional de secas y lluvias que afectan el proceso de reclutamiento de las especies de parásitos (Salgado-Maldonado, 1993), o con incrementos estacionales en la actividad trófica y reproductiva del hospedero, influenciados por las fluctuaciones temporales de la temperatura del agua (Jiménez, 2003).

Otros estudios sugieren que en climas tropicales las comunidades tienden a presentar una estructura estable a lo largo del tiempo (Pineda-López, 1994). Por lo tanto, aún no está claro si en los ambientes tropicales pueden registrarse cambios temporales en las comunidades de parásitos. Los datos obtenidos a lo largo de varios años, considerando 2 temporadas climáticas (secas y lluvias), permiten explorar este importante aspecto de la ecología de comunidades. El objetivo del presente estudio fue determinar la variación temporal en la estructura y composición de la comunidad de parásitos metazoarios de la charra C. trimaculatum en la laguna de Tres Palos, Guerrero.

\section{Materiales y métodos}

Un total de 231 ejemplares de C. trimaculatum, con longitudes de 11.1 a $19 \mathrm{~cm} \quad(15.1 \pm 1.49)$ fueron recolectados durante 4 temporadas climáticas (secas del 2000, 2001 y lluvias del 2000, 2002) en la laguna de Tres Palos, entre abril del 2000 y noviembre del 2002 (Cuadro 1). Los peces fueron sujetos a una necropsia completa incluyendo todos los órganos a excepción de la sangre y los huesos. Los parásitos metazoarios internos y externos que se recuperaron fueron procesados de acuerdo con los métodos descritos por Lamothe-Argumedo (1997) y VidalMartínez et al. (2001). Para determinar si el tamaño de muestra fue suficiente para recuperar la mayor proporción de especies de parásitos que caracterizó a la comunidad componente en cada una de las temporadas, se elaboraron curvas acumulativas de especies (Aho et al., 1991). Se depositaron muestras de la mayoría de los ejemplares identificados en la Colección Nacional de Helmintos del Instituto de Biología, UNAM.

Con base en sus estrategias de colonización, las especies de parásitos fueron clasificadas como: autogénicas, las que alcanzan la madurez en hospederos acuáticos, y alogénicas, las que presentan aves o mamíferos como hospederos definitivos (Esch et al., 1988). Se empleó además una segunda clasificación del hospedero (Rohde, 2005), organismos especialistas y generalistas. La variación en la composición de especies a lo largo del tiempo fue descrita empleando la prevalencia (porcentaje de hospederos infectados por una especie de parásito) y la abundancia promedio (número promedio de individuos parásitos por pez examinado), de acuerdo con lo sugerido por Bush et al. (1997). Para clasificar las especies como comunes o raras, se construyó una distribución de frecuencias con los valores de prevalencia de cada especie de parásito considerando todos los muestreos. Las diferencias entre los parámetros de infección entre temporadas fueron evaluadas mediante pruebas de independencia "G" (Sokal y Rohlf, 1998) en el caso de la prevalencia y Ancovas de una vía en el de la abundancia, considerando la talla como una covariable. La significancia considerada en los análisis fue de $\alpha=0.05$.

Las comunidades fueron analizadas en los niveles de componente y de infracomunidad (Holmes y Price, 1986). En el primero se consideró el número total de especies; el número total de individuos parásitos; el índice de ShannonWiener $(\mathrm{H})$, el cual es un índice de comunidad muestreada (expresa la uniformidad de las abundancias en una muestra), como medida de la diversidad; la equidad (uniformidad de las abundancias), y el índice de Berger-Parker (IBP), como medida de la dominancia numérica (Magurran, 1991). Para determinar diferencias significativas en los parámetros de comunidad componente entre temporadas climáticas 
Cuadro 1. Parámetros de infección de las especies parásitos registradas en el cíclido Cichlasoma trimaculatum de la laguna de Tres Palos, Guerrero

\begin{tabular}{|c|c|c|c|c|c|c|c|}
\hline Parásito & Hábitat & CNHE & N/temp. & Preval. & Total & Abundancia & $\begin{array}{l}\text { Rango de } \\
\text { intensidad }\end{array}$ \\
\hline \multicolumn{8}{|l|}{ Digenea } \\
\hline $\begin{array}{l}\text { Ascocotye (Phagicola) } \\
\text { longa }^{\mathrm{Al}} \text { (Ransom, 1920) }\end{array}$ & $\begin{array}{l}\text { Corazón, } \\
\text { mesenterio, hígado }\end{array}$ & 5986 & $\begin{array}{l}\text { 48/Sec00 } \\
\text { 72/Lluv00 } \\
\text { 63/Lluv02 }\end{array}$ & $\begin{array}{l}4.2 \\
2.8 \\
6.3\end{array}$ & $\begin{array}{l}2 \\
6 \\
8\end{array}$ & $\begin{array}{c}0.1 \\
0.1 \\
0.1 \pm 0.8\end{array}$ & $\begin{array}{l}2-2 \\
3-3 \\
1-3\end{array}$ \\
\hline $\begin{array}{l}\text { Austrodiplostomum } \\
\text { compactum }^{\text {Al }} \text { (Lutz, 1928) } \\
\text { Dubois, } 1970\end{array}$ & Ojos & 5987 & $\begin{array}{l}\text { 48/Sec00 } \\
\text { 72/Lluv00 } \\
\text { 63/Lluv02 }\end{array}$ & $\begin{array}{l}25.0 \\
37.5 \\
27.0\end{array}$ & $\begin{array}{c}7 \\
51 \\
59\end{array}$ & $\begin{array}{l}0.3 \pm 0.4 \\
0.7 \pm 0.6 \\
0.9 \pm 2.7\end{array}$ & $\begin{array}{l}1-2 \\
1-3 \\
1-10\end{array}$ \\
\hline $\begin{array}{l}\text { Cladocystis trifolium } \\
\text { (Braun, 1901) }\end{array}$ & Branquias & 4902 & $\begin{array}{l}\text { 72/Lluv00 } \\
\text { 48/Sec01 } \\
\text { 63/Lluv02 }\end{array}$ & $\begin{array}{c}4.2 \\
4.2 \\
25.4\end{array}$ & $\begin{array}{c}10 \\
3 \\
52\end{array}$ & $\begin{array}{l}0.1 \pm 4.0 \\
0.06 \\
0.8 \pm 6.4\end{array}$ & $\begin{array}{c}1-8 \\
1-2 \\
1-27\end{array}$ \\
\hline $\begin{array}{l}\text { Clinostomum } \\
\text { complanatum }^{\mathrm{Al}} \\
\text { (Rudolphi, 1814) }\end{array}$ & $\begin{array}{l}\text { Hígado, mesenterio, } \\
\text { músculo, cavidad } \\
\text { corporal }\end{array}$ & 4905 & $\begin{array}{l}\text { 48/Sec00 } \\
\text { 72/Lluv00 } \\
\text { 48/Sec01 } \\
\text { 63/Lluv02 }\end{array}$ & $\begin{array}{l}25.0 \\
25.0 \\
12.5 \\
14.3\end{array}$ & $\begin{array}{c}35 \\
129 \\
35 \\
163\end{array}$ & $\begin{array}{c}1.5 \pm 4.6 \\
1.8 \pm 8.1 \\
0.7 \pm 7.5 \\
2.6 \pm 47.2\end{array}$ & $\begin{array}{l}1-13 \\
1-30 \\
1-21 \\
1-144\end{array}$ \\
\hline $\begin{array}{l}\text { Crassicutis cichlasomae } \\
\text { (Manter, 1936) }\end{array}$ & Intestino & 4907 & $\begin{array}{l}\text { 48/Sec00 } \\
\text { 72/Lluv00 } \\
\text { 48/Sec01 } \\
\text { 63/Lluv02 }\end{array}$ & $\begin{array}{c}8.3 \\
36.1 \\
16.7 \\
9.5\end{array}$ & $\begin{array}{c}13 \\
567 \\
32 \\
17\end{array}$ & $\begin{array}{c}0.5 \pm 7.8 \\
7.9 \pm 40.1 \\
0.7 \pm 3.6 \\
0.3 \pm 2.1\end{array}$ & $\begin{array}{c}1-12 \\
1-207 \\
1-12 \\
1-7\end{array}$ \\
\hline $\begin{array}{l}\text { Posthodiplostomum } \\
\text { minimum Al (MacCallum, } \\
\text { 1921) Dubois, } 1936\end{array}$ & Músculo & 5988 & $\begin{array}{l}\text { 72/Lluv00 } \\
\text { 48/Sec01 } \\
\text { 63/Lluv02 }\end{array}$ & $\begin{array}{l}13.9 \\
16.7 \\
36.5\end{array}$ & $\begin{array}{c}35 \\
37 \\
129\end{array}$ & $\begin{array}{l}0.5 \pm 0.8 \\
0.8 \pm 1.4 \\
2.0 \pm 6.8\end{array}$ & $\begin{array}{l}2-5 \\
3-7 \\
1-27\end{array}$ \\
\hline $\begin{array}{l}\text { Pseudoacanthostomum } \\
\text { panamense Au (Caballero, } \\
\text { Bravo-Hollis y Grocott, } \\
\text { 1953) }\end{array}$ & $\begin{array}{l}\text { Músculo, pared del } \\
\text { intestino }\end{array}$ & & $\begin{array}{l}\text { 48/Sec01 } \\
63 / \text { Lluv02 }\end{array}$ & $\begin{array}{l}27.1 \\
15.9\end{array}$ & $\begin{array}{l}56 \\
72\end{array}$ & $\begin{array}{l}1.2 \pm 4.3 \\
1.1 \pm 9.6\end{array}$ & $\begin{array}{l}1-15 \\
1-30\end{array}$ \\
\hline \multicolumn{8}{|l|}{ Nematoda } \\
\hline Contracaecum sp. ${ }^{\mathrm{Al}}$ & $\begin{array}{l}\text { Mesenterio, } \\
\text { hígado, músculo }\end{array}$ & 4908 & $\begin{array}{l}\text { 48/Sec00 } \\
\text { 72/Lluv00 } \\
\text { 48/Sec01 } \\
\text { 63/Lluv02 }\end{array}$ & $\begin{array}{l}25.0 \\
11.1 \\
12.5 \\
63.5\end{array}$ & $\begin{array}{c}9 \\
29 \\
12 \\
305\end{array}$ & $\begin{array}{l}0.4 \pm 0.8 \\
0.4 \pm 4.3 \\
0.3 \pm 1.1 \\
4.8 \pm 8.2\end{array}$ & $\begin{array}{c}1-3 \\
1-14 \\
1-4 \\
1-39\end{array}$ \\
\hline \multicolumn{8}{|l|}{ Acantocephala } \\
\hline $\begin{array}{l}\text { Neoechinorhynchus } \\
\text { golvani }^{\text {Au }} \text { (Salgado- } \\
\text { Maldonado, 1978) }\end{array}$ & Intestino & 5989 & $\begin{array}{l}\text { 48/Sec00 } \\
\text { 72/Lluv00 } \\
\text { 48/Sec01 } \\
\text { 63/Lluv02 }\end{array}$ & $\begin{array}{l}4.2 \\
2.8 \\
2.1 \\
7.9\end{array}$ & $\begin{array}{c}2 \\
69 \\
1 \\
17\end{array}$ & $\begin{array}{c}0.1 \\
1.0 \pm 40.3 \\
0.02 \\
0.3 \pm 1.1\end{array}$ & $\begin{array}{c}2-2 \\
6-63 \\
1-1 \\
2-5\end{array}$ \\
\hline $\begin{array}{l}\text { Southwellina hispida }{ }^{\mathrm{Al}} \\
\text { (Van Cleave, 1916) }\end{array}$ & $\begin{array}{l}\text { Hígado, } \\
\text { mesenterio }\end{array}$ & 5990 & $\begin{array}{l}\text { 72/Lluv00 } \\
\text { 48/Sec01 } \\
\text { 63/Lluv02 }\end{array}$ & $\begin{array}{c}5.6 \\
8.3 \\
22.2\end{array}$ & $\begin{array}{c}8 \\
5 \\
50\end{array}$ & $\begin{array}{l}0.1 \pm 1.4 \\
0.1 \pm 0.5 \\
0.8 \pm 5.0\end{array}$ & $\begin{array}{c}1-4 \\
1-2 \\
1-19\end{array}$ \\
\hline \multicolumn{8}{|l|}{ Crustacea } \\
\hline Argulus sp. ${ }^{\mathrm{Au}}$ & Piel, aletas & & $\begin{array}{l}\text { 72/Lluv00 } \\
\text { 63/Lluv02 }\end{array}$ & $\begin{array}{l}4.2 \\
3.2\end{array}$ & $\begin{array}{l}3 \\
2\end{array}$ & $\begin{array}{l}0.04 \\
0.03\end{array}$ & $\begin{array}{l}- \\
-\end{array}$ \\
\hline Ergasilus sp. ${ }^{\mathrm{Au}}$ & Branquias & & $\begin{array}{l}\text { 48/Sec00 } \\
\text { 72/Lluv00 } \\
\text { 48/Sec01 } \\
\text { 63/Lluv02 }\end{array}$ & $\begin{array}{l}100 \\
100 \\
100 \\
100\end{array}$ & $\begin{array}{l}4244 \\
11715 \\
11017 \\
11963\end{array}$ & $\begin{array}{c}176.8 \pm 74.7 \\
162.7 \pm 89.2 \\
229.5 \pm 116.1 \\
189.9 \pm 78.7\end{array}$ & $\begin{array}{c}102-440 \\
89-698 \\
101-557 \\
65-525\end{array}$ \\
\hline
\end{tabular}

Estrategias de colonización: $\mathrm{Au}=$ autogénica, $\mathrm{Al}=$ alogénica. $\mathrm{CNHE}$ : Colección Nacional de Helmintos. $\mathrm{N}=$ número de hospederos examinados. Temp. = temporada climática, Sec $=$ Secas (diciembre-mayo), Lluv = lluvias (junio-noviembre). Preval. $=$ prevalencia de infección $(\%$ de hospederos infectados). Total = número total de individuos parásitos. Abundancia (número promedio de parásitos por hospedero examinado \pm desviación estándar). 
(secas y lluvias) se emplearon pruebas de Kruskal-Wallis. Las infracomunidades fueron descritas mediante el número promedio de especies de parásitos, el número promedio de individuos y el valor promedio del índice de diversidad de Brillouin $\left(\mathrm{H}^{\prime}\right)$, que es más sensible a los cambios en la abundancia de las especies raras (Krebs, 1999). Se emplearon Ancovas de una vía para determinar diferencias significativas en los parámetros entre temporadas de este nivel. Las correlaciones efectuadas se llevaron a cabo por medio del coeficiente por rangos de Spearman $\left(\mathrm{r}_{\mathrm{s}}\right)$ (Krebs, 1999).

\section{Resultados}

Se identificaron 12 especies de parásitos metazoarios en los 231 ejemplares de C. trimaculatum examinados durante los muestreos: 7 digéneos, 2 acantocéfalos, 1 nematodo y 2 crustáceos (Cuadro 1). Fueron recuperadas 8 especies de helmintos en forma larvaria de diversas estructuras, tales como el corazón, hígado, mesenterio, músculo, branquias y ojos; 7 de estas especies se clasificaron como alogénicas, y como autogénicas las 2 de crustáceos y 3 de helmintos (Crassicutis cichlasomae, Pseudoacanthostomum panamense y Neoechinorhynchus golvani (Cuadro 1). El digéneo $C$. cichlasomae y el acantocéfalo $N$. golvani fueron clasificados como especialistas, y las 11 especies restantes generalistas. La distribución de frecuencias de los valores de prevalencia de cada especie de parásito considerando todos los muestreos, permitió separarlos en 2: un grupo de especies comunes (prevalencia $>20 \%$ ) integrado por Ergasilus sp., Contracaecum sp., Austrodiplostomum compactum, C. cichlasomae, Posthodiplostomum minimum y Clinostomum complanatum, y otro grupo de especies raras (prevalencia $<20 \%$ ).

De las especies de parásitos clasificadas como comunes, 4 registraron variación temporal en ambos parámetros de infección (C. cichlasomae y Contracaecum sp.), o en solo uno de ellos (C. complanatum y Ergasilus sp.). El porcentaje de infección más alto de C. cichlasomae se registró en la temporada de lluvias del año $2000(\mathrm{G}=$ 28.01, $p<0.05)$; el de C. complanatum en secas de ese mismo año $(\mathrm{G}=8.10, p<0.05)$, y el de Contracaecum sp. en lluvias del $2002(\mathrm{G}=64.02, p<0.05)$. En tanto que la abundancia de $C$. cichlasomae fue mayor en lluvias del 2000 (Ancova F = 3.55, $p<0.05$ ); la de Contracaecum sp. en lluvias del 2002 (Ancova $\mathrm{F}=3.72, p<0.05$ ), y la de Ergasilus sp. en secas del 2001 (Ancova $\mathrm{F}=4.18, p<$ $0.05)$.

Con base en las curvas acumulativas de especies construidas para cada una de las temporadas se estableció que más del $90 \%$ de las especies comunes (6 especies) pudieron ser recuperadas con sólo examinar 40 peces (Fig. 1). No se registró correlación entre el tamaño de las muestras y los parámetros considerados en ninguno de los 2 niveles $(p>0.05)$. El número de especies de parásitos recuperado en los muestreos varió entre 7 y 12 (Cuadro 2), siendo significativamente más alto en la temporada de lluvias del 2002 (Kruskal-Wallis $\mathrm{H}=8.76, p<0.05$ ). Mientras que el total de individuos fluctuó de 4312 (secas del 2001) a 12837 individuos (lluvias del 2002), y fue más alto en las 2 temporadas de lluvias (Kruskal-Wallis $\mathrm{H}=10.25, p<$ 0.05). Ergasilus sp. dominó numéricamente en todas las temporadas climáticas (Cuadro 2); en tanto que entre las especies de helmintos, C. cichlasomae y C. complanatum fueron las que presentaron mayor dominancia.

La diversidad (índice de Shannon-Wiener) fluctuó entre 0.15 y 0.55 bits/ind; sin embargo, cuando la especie numéricamente dominante (Ergasilus sp.) fue eliminada del análisis, los valores variaron entre 1.97 y 2.73 bits/ ind (Cuadro 2). La comparación entre temporadas indicó que la diversidad fue significativamente más alta en la de lluvias del 2002 (Kruskal-Wallis $\mathrm{H}=18.38, p<0.05$ ).

Todos los ejemplares de $C$. trimaculatum examinados resultaron infectados; el intervalo de especies de parásitos por hospedero infectado fue de 1 a 7 . El $12 \%$ de los peces estuvo parasitado por una sola especie, mientras que el $88 \%$ registró infecciones mixtas. El $45 \%$ presentó 2 especies, el $41 \%$ de 3 a 5 y sólo el $2 \%$ presentó de 6 a 7 especies distintas. Considerando todos los muestreos, la longitud total de los hospederos fue correlacionada positivamente con el número de especies $\left(\mathrm{r}_{\mathrm{s}}=0.27, p<0.05, n=231\right) \mathrm{y}$ el número de individuos $\left(\mathrm{r}_{\mathrm{s}}=0.32, p<0.05, n=231\right)$.

El número promedio de especies de parásitos fue de $1.92 \pm 0.7$ a $3.32 \pm 1.3$, siendo significativamente más alto en la temporada de lluvias del 2002 (Ancova $\mathrm{F}=28.8, p<$ 0.05). El número promedio de individuos parásitos varió entre $175.3 \pm 92.7$ y $233.3 \pm 117.1$ y fue mayor en secas del 2001 (Ancova $\mathrm{F}=3.95, p<0.05$ ). La diversidad promedio (índice de Brillouin) varió entre $0.14 \pm 0.2$ y $0.40 \pm 0.3$, siendo más alta en las 2 temporadas de lluvias (Ancova $\mathrm{F}$ $=12.54, p<0.05)$.

\section{Discusión}

Todas las especies identificadas en este estudio han sido previamente registradas en la laguna de Tres Palos (Violante-González y Méndez, 2003; Garrido-Olvera et al., 2004; Violante-González et al., 2007) o en la de Coyuca (Violante-González y Aguirre-Macedo, 2007).

El bajo número de especies intestinales recuperadas de $C$. trimaculatum en Tres Palos (C. cichlasomae y $N$. golvani) contrasta con lo observado en las comunidades de 


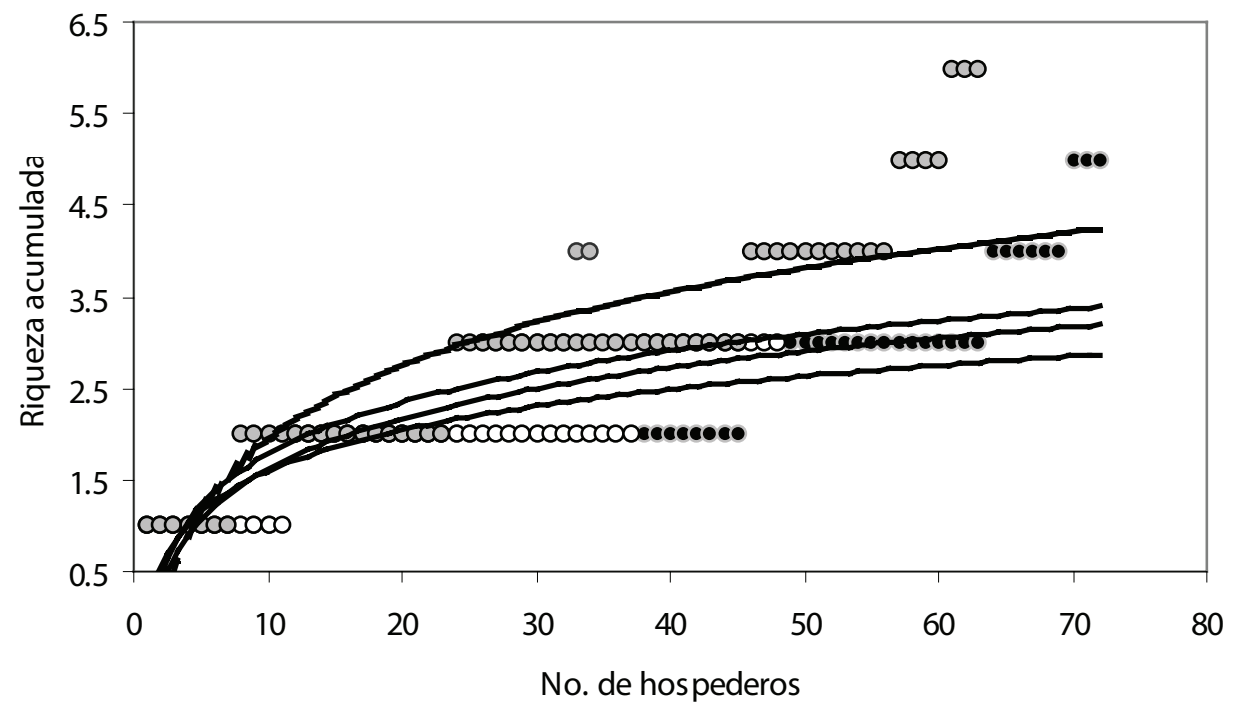

Figura 1. Curvas acumulativas de especies por temporada climática. $\mathrm{a}=$ secas $00(n=48), \mathrm{b}=1$ luvias $00(n=72), \mathrm{c}=\operatorname{secas} 01(n=$ 48), $\mathrm{d}=$ lluvias $02(n=63)$.

Cuadro 2. Características de las comunidades componentes y las infracomunidades de parásitos de Cichlasoma trimaculatum en la laguna de Tres Palos, Guerrero, durante cuatro temporadas climáticas

\begin{tabular}{cllllllllllllll}
\hline \multicolumn{10}{c}{ Comunidad componente } & \multicolumn{1}{c}{ Infracomunidad } \\
\hline Temp. & $\begin{array}{l}\text { Núm. } \\
\text { hosp. }\end{array}$ & $\begin{array}{l}\text { Núm. } \\
\text { desp. }\end{array}$ & $\begin{array}{l}\text { Núm. } \\
\text { parásit. }\end{array}$ & IBP & EP & $H$ & Equidad & $H^{*}$ & Equidad ${ }^{*}$ & $\begin{array}{l}\text { Núm. } \\
\text { promedio } \\
\text { de especies }\end{array}$ & $\begin{array}{l}\text { Núm. } \\
\text { promedio de } \\
\text { individuos }\end{array}$ & $\mathrm{H}^{\prime}$ \\
Secas 00 & 48 & 7 & 4312 & 0.93 & Erga & 0.15 & 0.05 & 1.97 & 0.76 & $1.92 \pm 0.7$ & $179.7 \pm 74.4$ & $0.16 \pm 0.2$ \\
Lluvias 00 & 72 & 11 & 12622 & 0.98 & Erga & 0.51 & 0.15 & 1.88 & 0.57 & $2.43 \pm 0.9$ & $175.3 \pm 92.7$ & $0.4 \pm 0.3$ \\
Secas 01 & 48 & 9 & 11198 & 0.92 & Erga & 0.16 & 0.05 & 2.43 & 0.81 & $2 \pm 0.7$ & $233.3 \pm 117.1$ & $0.14 \pm 0.2$ \\
Lluvias 02 & 63 & 12 & 12837 & 0.98 & Erga & 0.55 & 0.15 & 2.73 & 0.79 & $3.32 \pm 1.3$ & $203.8 \pm 82.7$ & $0.39 \pm 0.3$ \\
\hline
\end{tabular}

IBP = índice de Berger-Parker, EP = especie dominante, Erga = Ergasilus sp, H = índice de diversidad de Shannon-Wiener; * = valores obtenidos después de eliminar a la especie dominante, $\mathrm{H}^{\prime}=$ índice de diversidad de Brillouin.

parásitos de cíclidos del sureste de México, donde se han registrado comunidades intestinales de 5 especies en el cíclido Thorichthys helleri en El Espino, Tabasco (SalgadoMaldonado et al., 2005b) y hasta 18 en Cichlasoma (Theraps) synspilum en la laguna de Términos, Campeche (Vidal-Martínez y Kennedy, 2000).

La comunidad de parásitos de C. trimaculatum sólo incluyó 2 helmintos especialistas, el digéneo C. cichlasomae y el acantocéfalo $N$. golvani; aunque el digéneo C. trifolium también puede considerarse como un especialista, ya que únicamente se ha registrado en cíclidos (Vidal-Martínez et al., 2001; Salgado-Maldonado et al., 2005a, b).

En términos generales, los cíclidos se caracterizan por su estrecha asociación con las especies de digéneos C. cichlasomae, Oligogonotylus manteri y Genarchella isabellae, los nematodos Procamallanus (Spirocamallanus) rebecae y Rhabdochona kidderi y el acantocéfalo Neoechinorhynchus golvani (Salgado-Maldonado et al., 2005a). La razón por la cual C. trimaculatum no presenta otras especies de este conjunto bien definido de helmintos, es que no existen otros representantes nativos de la familia Cichlidae, con los cuales pueda intercambiar especialistas, por lo que su comunidad está constituida en más del $80 \%$ (10/12) por generalistas. Esta afirmación la respaldan los resultados obtenidos por Salgado-Maldonado et al. (2001), quienes informan que la comunidad de parásitos del cíclido nativo Cichlasoma istlanum en el río Balsas (Guerrero) estuvo constituida por 4 especies, registrando como único especialista el acantocéfalo $N$. golvani.

Otro resultado importante es que no se encontró ninguna 
especie de monogéneo en los hospederos examinados; en cambio Ergasilus sp. fue el ectoparásito que dominó numéricamente la comunidad durante todos los muestreos. En este sentido, Sciadicleithrum es un género de monogéneos altamente específico de cíclidos neotropicales, del cual existen 4 especies en el sureste de México $(S$. bravohollisae, S. meeki, S. mexicanum y S. splendida) que infectan unas 36 especies de cíclidos (MendozaFranco et al., 1999). No obstante que Sciadicleithrum mexicanum se ha identificado en organismos cultivados de C. trimaculatum, así como en poblaciones silvestres del río Papagayo (datos no publicados), este monogéneo no estuvo presente en la laguna de Tres Palos. Su ausencia se puede atribuir a que es una especie dulceacuícola que habita generalmente en ríos (Mendoza-Franco et al., 2000) y podría no prosperar en las lagunas costeras de Guerrero, que son ambientes salobres inestables y altamente contaminados, donde es posible que sea reemplazada por el copépodo Ergasilus sp. (Violante-González, 2006).

De las 6 especies clasificadas como comunes, 4 presentaron una clara variación temporal en su dinámica de infección a lo largo del tiempo. Algunas fueron más prevalentes o abundantes en la temporada de secas ( $C$. complanatum y Ergasilus sp.), en tanto que otras en la de lluvias (C. cichlasomae y Contracaeucum sp.); sin embargo, no se observó un patrón claro; por ejemplo, sólo el digéneo C. cichlasomae registró una mayor prevalencia y abundancia en la misma temporada (lluvias del 2000, Cuadro 1); no obstante, 2 años después presentó infecciones bajas en igual época, lo que sugiere que los niveles de infección a lo largo del tiempo son poco predecibles y es posible atribuirlas a las condiciones ambientales que suelen variar de un año a otro (Violante-González, 2006); esto es, la precipitación pluvial así como los periodos de estiaje pueden ser distintos entre años, por lo que la influencia de estos factores sobre las poblaciones de parásitos o de sus hospederos intermediarios no sería la misma.

La comunidad componente se caracterizó por ser pobre en número de especies (12), con diversidad baja y altamente dominada, cuando se le compara con algunas comunidades de cíclidos del sureste como las de Cichlasoma (Nandopsis) urophthalmus (38 especies), Parachromis managuensis (34) y T. hellerin (32) (Salgado-Maldonado et al., 2005b). Sin embargo, la riqueza fue similar a la observada para otras comunidades componentes de peces dulceacuícolas tropicales (Choudhury y Dick, 2000; Jiménez, 2003; Salgado-Maldonado et al., 2004a, b).

El mayor número de helmintos en forma larvaria que presentó la comunidad componente (8/10) sugiere que C. trimaculatum desempeña un papel importante como un hospedero intermediario (Zander et al., 1999) para las especies de helmintos parásitos en la laguna de Tres Palos.
Por otra parte, la gran abundancia de aves ictiófagas que habita de manera permanente en los márgenes de la laguna contribuye de manera importante en la composición de la comunidad de parásitos de este cíclido, al actuar como hospederos definitivos y dispersores de la mayor parte de sus especies de parásitos. Esto se ve reflejado por el mayor número de especies alogénicas recuperadas (7/10 helmintos), las cuales maduran en algunas de las especies más abundantes, como Phalacrocorax olivaceus y Casmerodius albus (datos no publicados).

La gran abundancia de digéneos que incluyó la comunidad (7/10 helmintos) se atribuye al hábito alimenticio y a la preferencia que tiene este cíclido por las raíces de los manglares como hábitat (Violante-González, 1995), lo cual lo pone en contacto con caracoles, que actúan como primeros hospederos intermediarios de este grupo de helmintos. La dieta omnívora de C. trimaculatum (Yáñez-Arancibia, 1978) también se considera un factor importante que contribuye a la adquisición de un mayor número de especies de digéneos (Violante-González, 2006). Los digéneos son además, el grupo numéricamente dominante en muchas comunidades de parásitos de peces dulceacuícolas en México, y su abundancia ha sido relacionada con la poca profundidad y la alta productividad de las lagunas costeras (Salgado-Maldonado y Kennedy, 1997).

Las infracomunidades presentaron el mismo patrón que la comunidad componente; esto es, fueron pobres en número de especies, altamente dominadas y poco diversas, coincidiendo con lo observado en infracomunidades de peces dulceacuícolas (Kennedy et al., 1986; Kennedy, 1990). Las correlaciones positivas significativas que se registraron entre la longitud total de los hospederos y el número promedio de especies y de individuos parásitos indica que los peces de mayor tamaño albergaron infracomunidades con un número superior de especies y de individuos, lo que se atribuye a que los hospederos grandes (más viejos) han tenido más tiempo que los pequeños (más jóvenes) para acumular parásitos (Poulin, 2000; Bush et al., 2001).

Los principales factores que estructuran las infracomunidades de parásitos fueron los mismos que estructuran la comunidad componente (Holmes, 1990; Zander, 2004); es decir, el comportamiento alimenticio y la variación en la disponibilidad de estadios larvales, factores que son influenciados por las temporadas de secas y lluvias. Estos factores, estructuran las infracomunidades a partir de la acumulación de cercarias de vida libre (A. compactum, C. complanatum y $P$. minimum), o de la ingestión de presas infectadas, como caracoles (C. cichlasomae) o crustáceos (Contracaecum sp. y Southwellina hispida). La estructuración se completa a partir de la adquisición de 
algunas especies raras (Ascocotye (P.) longa, C. trifolium, P. panamense y N. golvani).

Las infracomunidades, al igual que la comunidad componente, presentaron cambios temporales en su estructura, los cuales fueron atribuidos a cambios en la dinámica de infección de algunas especies comunes. Sin embargo, no se observó un patrón temporal de estructuración definido, ya que los cambios en la estructura no fueron constantes entre años, por lo que pueden ser poco predecibles, como ha sido sugerido para otras infracomunidades de parásitos de peces dulceacuícolas (Poulin y Valtonen, 2002; Vidal-Martínez y Poulin, 2003).

Como conclusión, podemos decir que la comunidad componente de C. trimaculatum, es distinta a la observada en otras comunidades de parásitos de cíclidos del sureste de México. Ésta se caracteriza por un mayor número de parásitos generalistas y pocos especialistas, lo cual se atribuye a que es la única especie de cíclido nativo en la localidad, por lo que no existe la posibilidad de intercambio de especialistas con otros miembros de la misma familia. Además, su comunidad presenta variaciones en la estructura a lo largo del tiempo, como resultado del efecto de los factores ambientales sobre la dinámica de infección de sus especies de parásitos, aunque los cambios en la estructura pueden ser poco predecibles debido a la gran inestabilidad ambiental que se observa en la localidad.

\section{Agradecimientos}

Al Sistema de Investigación Benito Juárez (SIBEJ), por el financiamiento del proyecto de investigación 19990502017; a los estudiantes de la Unidad Académica de Ecología Marina (UAG) por el apoyo en el trabajo de campo y laboratorio; al Dr. Guillermo Salgado-Maldonado y al Dr. David Osorio-Sarabia, por su ayuda en la identificación de algunas especies de parásitos. Agradecemos también a los revisores anónimos, cuyos comentarios y sugerencias contribuyeron a mejorar sustancialmente este manuscrito.

\section{Literatura citada}

Aho, J. M., A. O. Bush y R. W. Wolfe. 1991. Helminth parasites of bowfin (Amia calva) from South California. Journal of the Helminthological Society of Washington 58:171-175.

Bush, A. O., K. D. Lafferty, J. M. Lotz y A. W. Shostak. 1997. Parasitology meets ecology on its own terms: Margolis et al. revisited. Journal of Parasitology 83:575-583.

Bush, A. O., J. C. Fernández, G. W. Esch y J. R. Seed. 2001. Parasitism: The diversity and ecology of animal parasites. Cambridge University Press. 566 p.
Choudhury, A. y T. A. Dick. 2000. Richness and diversity of helminth communities in tropical freshwater fishes: empirical evidence. Journal of Biogeography 27:935-956.

Esch, G. W., C. R. Kennedy, A. O. Bush y J. M. Aho. 1988. Patterns in helminth communities in freshwater fish in Great Britain: alternative strategies for colonization. Parasitology 96:519-532.

Garrido-Olvera, L., L. García-Prieto y B. Mendoza-Garfias. 2004. Helminth parasites of the fat sleeper, Dormitator latifrons (Richardson, 1844) from Tres Palos Lagoon, Guerrero, Mexico. American Midland Naturalist 151:163-169.

Holmes, J. C. y P. W. Price. 1986. Communities of parasites. In Community ecology: patterns and process, cap. 9, J. Kikkawa y D. J. Anderson (eds.). Chapman and Hall, London. 335 p.

Holmes, J. C. 1990. Helminth communities of marine fishes. In Parasite communities: patterns and process, G. W. Esch, A. O. Bush y J. M. Aho (eds.) Chapman and Hall, London. p.101-130.

Jiménez, M. I. 2003. Estructura temporal de las comunidades de parásitos metazoarios en poblaciones de cíclidos silvestres (Cichlasoma urophthalmus) e introducidos en Mitza, Yucatán: un enfoque experimental. Tesis, doctorado CINVESTAV- IPN, Unidad Mérida, Yucatán 174 p.

Kennedy, C. R., A. O. Bush y J. M. Aho. 1986. Patterns in helminth communities: Why are bird and fishes different?. Parasitology 93:205-215.

Kennedy, C. R. 1990. Helminth communities in freshwater fish: structured communities or stochastic assemblages? In Parasite communities: patterns and processes. G. W. Esch, A. O. Bush y J. M. Aho (eds.). Chapman and Hall, London. p. 131-156.

Krebs, C. J. 1999. Ecological methodology. Harper Collings, $654 \mathrm{p}$.

Lamothe-Argumedo, R. 1997. Manual de técnicas para preparar y estudiar los parásitos de animales silvestres. AGT, México, D.F. 43 p.

Magurran, A. 1991. Ecological diversity and its measurement. Princeton University Press, New Jersey. 178 p.

Mendoza-Franco, E. F, T. Scholz, C. Vivas-Rodríguez y J. Vargas-Vázquez. 1999. Monogeneans of freshwater fishes from cenotes (sinkholes) of the Yucatán Peninsula, Mexico. Folia Parasitologica 46:267-273.

Mendoza-Franco, E., V. M. Vidal-Martínez, M. L. AguirreMacedo, R. Rodríguez-Canul, y T. Scholz. 2000. Species of Sciadicleithrum (Dactylogyridae: Ancyrocephalinae) of cichlid fishes from southeastern México and Guatemala: New morphological data and host and geographical records. Comparative Parasitology 67:85-91.

Pineda-López, R. 1994. Ecology of the helminth communities of cichlid fish in the flood plains of Southeastern México. Ph. D.- Thesis, University of Exeter. 236 p.

Poulin, R. 2000. Variation in the intraspecific relationship between fish length and intensity of parasitic infection: biological and statistical causes. Journal of Fish Biology 56:123-137.

Poulin. R. y E. T. Valtonen. 2002. The predictability of helminth community structure in space: a comparison of fish populations from adjacent lakes. International Journal for Parasitology 10:1235-1243. 
Rohde, K. 2005. Ecology of marine parasites. CSIRO, Melbourne and CABI, Wallingford, Oxon, $565 \mathrm{p}$.

SAGARPA (Secretaría de Agricultura, Ganadería, Recursos pesqueros y Acuicultura). 2000. Informe anual de la Dirección General de Estudios sobre Producción Pesquera del Estado de Guerrero, Chilpancingo, México.

Salgado-Maldonado, G. 1993. Ecología de helmintos parásitos de Cichlasoma urophthalmus (Günter) (Pisces: Cichlidae) en la Península de Yucatán, México. Tesis doctorado CINVESTAV-IPN, Unidad Mérida, Yucatán.

Salgado-Maldonado, G. y C. R. Kennedy. 1997. Richness and similarity of helminth communities in the tropical fish Cichlasoma urophthalmus from the Yucatan Peninsula, Mexico. Parasitology 114:581-590.

Salgado-Maldonado, G., R. Pineda-López, V. M. Vidal-Martínez y C. R. Kennedy. 1997. A checklist of metazoan parasites of cichlid fish from México. Journal of the Helminthological Society of Washington 64:195-207.

Salgado-Maldonado, G., G. Cabañas-Carranza, J. M. CaspetaMandujano, E. Soto-Galera, E. Mayén-Peña, D. Brailovski y R. Báez-Valé. 2001. Helminth parasites of freshwater fishes of the Balsas river drainage basing of southwestern Mexico. Comparative Parasitology 68:196-203.

Salgado-Maldonado, G., G. Cabañas-Carranza, E. Soto-Galera, R. Pineda-López, J. M. Caspeta-Mandujano, E. AguilarCastellanos y N. Mercado-Silva. 2004a. Helminth parasites of freshwater fishes of the Pánuco river basin, east central Mexico. Comparative Parasitology 71:190-202.

Salgado-Maldonado, G., N. Mercado-Silva, G. CabañasCarranza, J. M. Caspeta-Mandujano, R. Aguilar-Aguilar y L. I. Iñíguez-Dávalos. 2004b. Helminth parasites of freshwater fishes of the Ayuquila river, Sierra de Manatlán Biosphere Reserve, west central Mexico. Comparative Parasitology 71:67-72.

Salgado-Maldonado, G., R. Aguilar-Aguilar, G. CabañasCarranza, E. Soto-Galera y C. Mendoza-Palmero. 2005a. Helminth parasites in freshwater fish from the Papaloapan river basin, México. Parasitology Research 96:69-89.

Salgado-Maldonado, G., R. Pineda-López, L. García-Magaña, S. López-Jiménez, V. M. Vidal-Martínez y M. L. AguirreMacedo. 2005b. Helmintos parásitos de peces dulceacuícolas. In Biodiversidad del estado de Tabasco, J. Bueno, F. Álvarez y S. Santiago (eds.). Instituto de Biología, UNAMCONABIO, México, D.F. 386 p.

Salgado-Maldonado, G. 2006. Checklist of helminth parasites of freshwater fishes from Mexico. Zootaxa 1324:1-357

Sokal, R. R. y F. J. Rohlf. 1998. Biometry, segunda edición. Freeman, San Francisco, California. 859 p.

Vidal-Martínez, V. M. 1995. Processes structuring the helminth communities of native cichlid fishes from Southern México.
Ph. D. Thesis. University of Exeter, Devon. 163 p.

Vidal-Martínez, V. M., C. R. Kennedy y M. L. Aguirre-Macedo. 1998. The structuring process of the macroparasite community of an experimental population of Cichlasoma urophthalmus through time. Journal of Helminthology 72:199-207.

Vidal-Martínez, V. M y C. R. Kennedy. 2000. Potential interactions between the intestinal helminthes of the cichlid fish Cichlasoma synspilum from Southeastern Mexico. Journal of Parasitology 86:691-695.

Vidal-Martínez, V. M., M. L. Aguirre-Macedo, T. Scholz, D. González-Solís y E. F. Mendoza Franco. 2001. Atlas of helminth parasites of Cichlid fish of Mexico. Academy of Sciences of the Czech Republic, Prague. 165 p.

Vidal-Martínez, V. M. y R. Poulin. 2003. Spatial and temporal repeatability in parasite community structure of tropical fish hosts. Parasitology 127:387-398.

Violante-González, J. 1995. Contribución al conocimiento de la biología de la mojarra nativa Cichlasoma trimaculatum (Günter, 1868), en la laguna de Tres Palos Gro., México y determinación del desarrollo larvario y requerimientos proteicos en condiciones de laboratorio. Tesis maestría FACIMAR, Universidad de Colima, Colima. 63 p.

Violante-González, J. y A. Méndez. 2003. Comunidad de parásitos metazoarios de Dormitator latifrons (popoyote), en dos lagunas costeras del estado de Guerrero, México. Ciencia. Universidad Autónoma de Guerrero 11:15-17.

Violante-González, J. 2006. Comunidades de parásitos metazoarios de peces, en dos lagunas costeras del Estado de Guerrero, México. Tesis, doctorado CINVESTAV- IPN, Mérida,Yucatán. 155 p.

Violante-González, J. y M. L. Aguirre-Macedo. 2007. Metazoan parasites of fishes from Coyuca Lagoon, Guerrero, Mexico. Zootaxa 1531:39-48.

Violante-González, J., M. L. Aguirre-Macedo y E. F. MendozaFranco. 2007. A checklist of metazoan parasites of fish from Tres palos Lagoon, Guerrero, Mexico. Parasitology Research 102:151-161.

Yáñez-Arancibia, A. 1978. Taxonomía, ecología y estructura de las comunidades de peces en lagunas costeras con bocas efímeras del Pacífico de México, Publicaciones Especiales 2. Centro de Ciencias del Mar y Limnología, Universidad Autónoma de México, México, D.F. 306 p. .

Zander, C. D., L. W. Reimer y K. Barz. 1999. Parasite communities of the Salzhaff (Northwest Mecklenburg, Baltic Sea). I. Structure and dynamics of communities of littoral fish, especially small-sized fish. Parasitology Research 85:356-372.

Zander, C. D. 2004. Four-year monitoring of parasite communities in gobiid fishes of the south-western Baltic. II. Infracommunity. Parasitology Research 93:17-29. 